\title{
Verification of Reliability and Validity of the Feeding and Swallowing Scale for Premature Infants (FSSPI)
}

\author{
Chang Won Moon, MD, Han geul Jung, MS, Hee Jung Cheon, MS, Su Mi Oh, MS, \\ Young Ok Ki, MS, Jeong-Yi Kwon, MD, PhD
}

Department of Physical and Rehabilitation Medicine, Samsung Medical Center, Sungkyunkwan University School of Medicine, Seoul, Korea

\begin{abstract}
Objective To propose a new scale, the Feeding and Swallowing Scale for Premature Infants (FSSPI), based on videofluoroscopic swallowing study (VFSS) findings and to verify the reliability and validity of the FSSPI.

Methods One hundred thirty preterm infants who had undergone VFSS were enrolled in this retrospective study. The FSSPI was developed by referring to the Baby Regulated Organization of Subsystems and Sucking approach. The FSSPI score for each VFSS video was evaluated by a physiatrist as well as by three experienced speechlanguage pathologists. To verify the reliability of the FSSPI, the inter-evaluator and intra-evaluator associations for the FSSPI scores were analyzed. To verify the validity of the FSSPI, the association between FSSPI scores and clinical characteristics including prognosis-related factors was analyzed.

Results The mean gestational age was $27.3 \pm 2.8$ weeks. The FSSPI showed a high degree of both intra-rater reliability and inter-rater reliability. Also, there was a significant negative correlation between the FSSPI score and corrected age (CA) at the time of performing VFSS. Further, a significant positive correlation was observed between the FSSPI score and CA at the time of achieving full oral feeding. A significant negative correlation was observed between the FSSPI score and weight gain, between the 1st and 2nd month after birth, and between the 2nd and 3rd month after birth, respectively.

Conclusion In this study, we proposed a new clinical scale using VFSS to reflect the development of feeding and swallowing skills in preterm infants. Further, we verified the reliability and validity of the scale.
\end{abstract}

Keywords Premature, Fluoroscopy, Dysphagia, Feeding, Scales

Received August 22, 2016; Accepted December 22, 2016

Corresponding author: Jeong-Yi Kwon

Department of Physical and Rehabilitation medicine, Samsung Medical Center, Sungkyunkwan University School of Medicine, 81 Irwon-ro, Gangnamgu, Seoul 06351, Korea. Tel: +82-2-3410-2818, Fax: +82-2-3410-0052, E-mail: jeongyi.kwon@samsung.com

ORCID: Chang Won Moon (http://orcid.org/0000-0003-4096-7470); Han geul Jung (http://orcid.org/0000-0002-7530-1372); Hee Jung Cheon (http:// orcid.org/0000-0002-3889-2900); Su Mi Oh (http://orcid.org/0000-0002-9117-0851); Young Ok Ki (http://orcid.org/0000-0002-3763-2250); Jeong-Yi Kwon (http://orcid.org/0000-0003-2011-8834).

(c) This is an open-access article distributed under the terms of the Creative Commons Attribution Non-Commercial License (http://creativecommons.org/ licenses/by-nc/4.0) which permits unrestricted noncommercial use, distribution, and reproduction in any medium, provided the original work is properly cited. Copyright $\odot 2017$ by Korean Academy of Rehabilitation Medicine 


\section{INTRODUCTION}

In infants born preterm, one of the greatest concerns for discharge from the neonatal intensive care unit to their home is maturation of oral feeding skills. The risk for developing feeding complications in preterm infants is high due to weakness of the oral and laryngeal musculature needed for sucking and safe swallowing, as well as immaturity of neural substrates to coordinate the suckswallow-breathe pattern [1-3].

The cost of treating feeding disorders in infancy has become a greater concern in recent years due to the rise in premature births [4].

Early feeding skills during infancy include the infant's ability to engage and remain engaged in physiologically and behaviorally challenging tasks, organize the oromotor function, coordinate breathing with swallowing to avoid prolonged apnea or aspiration of fluids, and regulate the depth and frequency of breathing to maintain physiologic stability [5].

To determine whether an infant is able to feed successfully, one must begin with accurate assessment of feeding and swallowing skills. Thorough understanding of oral feeding and swallowing skills aids the clinician in assigning a prognosis of feeding outcomes in preterm infants. Although videofluoroscopic swallowing study (VFSS) is regarded as the gold standard for the evaluation of dysphagia [6], the traditional interpretation of VFSS is mainly focused on anatomical and physiological abnormalities during swallowing [7] and is less informative for understanding the early development of feeding and swallowing skills during infancy. On the other hand, the Baby Regulated Organization of Subsystems and Sucking (BROSS) approach is typically used for evaluation of the developmental stage of feeding in the clinic [8].

In this study, we proposed a new scale, the Feeding and Swallowing Scale for Premature Infants (FSSPI), based on VFSS findings and the BROSS approach to reflect the development of feeding and swallowing skills. Thus, the objective of this study is to verify the validity and reliability of the FSSPI.

\section{MATERIALS AND METHODS}

This study was performed retrospectively. The study protocol was approved by the Institutional Review Board of Samsung Medical Center, a tertiary medical center in Seoul, Korea (No. 2014-09-061). A waiver of consent was granted for a chart review without patient contact.

\section{Development of the FSSPI}

One physiatrist and three speech-language pathologists with more than 5 years' experience of working in the dysphagia clinic developed this scale, which is in reference to the BROSS approach. The BROSS approach consists of six consecutive developmental levels of feeding. These include non-nutritive sucking, obligatory phase, alternating phase, intermittent sucking phase, coordinated phase, and integrated phase. In the obligatory phase, infants suck in 10- to 20 -suck bursts without breathing. As the infants mature, they show an alternating pattern of sucking and breathing. This means that they suck for a burst of 3 to 5 sucks and alternate with breathing, albeit tachypneically. In this phase, initially there are frequently longer sucking bursts accompanied by mild desaturation, with recovery during the tachypneic catch up. In the next intermittent sucking phase, infants take brief catch breaths once every 2 to 3 sucks, and longer sucking bursts appear with the catch breaths imbedded. The hallmark of the coordinated phase is when the infant develops a mature and coordinated sucking pattern with sucking bursts of 20 to 30 sucks, seamlessly integrating breathing with sucking and swallowing. In this phase, many infants have a sucking-swallowing-breathing (SSB) rhythm (1:1:1), and most babies develop their own coordinated pattern with modulated suction and expression. This phase is typically observed after infants' transition from the neonatal intensive care unit to home. The integrated phase is described as having full coordination of sucking/swallowing and breathing without increased work of breathing or tachypnea, clear demands to be fed and enjoyment of eating, and unique characteristics of social interactions between the baby and the primary caregiver.

The FSSPI consists of the following six items (Table 1): (1) non-nutritive sucking, (2) nutritive sucking, (3) sucking pattern, (4) sucking burst, (5) developmental stage of SSB, and (6) regularity of rhythm. Sucking patterns are classified as immature, transitional, and mature patterns, which are in reference to the Neonatal Oral-Motor Assessment Scale (NOMAS) [9]. An immature sucking pattern involves a short burst of 3 to 5 sucks. A transitional sucking pattern involves a medium burst of 5 to 10 sucks, 
Table 1. The Feeding and Swallowing Scale for Premature Infants (FSSPI)

\begin{tabular}{|c|c|c|c|c|c|c|c|}
\hline $\begin{array}{l}\text { Scale } \\
\text { score }\end{array}$ & NNS & NS & $\begin{array}{l}\text { Sucking } \\
\text { pattern }\end{array}$ & Burst & $\begin{array}{l}\text { SSB developmental } \\
\text { stage }\end{array}$ & Rhythm & Assessment \\
\hline 8 & No & No & - & No & - & - & No NNS \& NS \\
\hline 7 & Yes & No & - & No & NNS & - & NNS only \\
\hline 6 & Yes & Yes & - & No & - & - & Incomplete SS coordination \\
\hline 5 & Yes & Yes & - & Yes & Obligatory phase $^{\mathrm{d})}$ & - & $\begin{array}{l}\text { SS coordination without } \\
\text { breathing }\end{array}$ \\
\hline 4 & Yes & Yes & Immature $^{\text {a) }}$ & Yes & Alternating phase ${ }^{\mathrm{e})}$ & Irregular & Alternating immature \\
\hline 3 & Yes & Yes & Transitional $^{\mathrm{b})}$ & Yes & Alternating phase & Irregular & Alternating transitional \\
\hline 2 & Yes & Yes & Mature $^{c)}$ & Yes & Intermittent phase ${ }^{\mathrm{f})}$ & Irregular & Intermittent \\
\hline 1 & Yes & Yes & Mature & Yes & Coordinated phase $^{\mathrm{g})}$ & Irregular & $\begin{array}{l}\text { Coordinated SSB but } \\
\text { irregular rhythm }\end{array}$ \\
\hline 0 & Yes & Yes & - & Yes & Integrated phase $\mathrm{e}^{\mathrm{h})}$ & Regular & $\begin{array}{l}\text { Coordinated SSB with } \\
\text { regular rhythm }\end{array}$ \\
\hline
\end{tabular}

NNS, non-nutritive sucking; NS, nutritive sucking; SSB, sucking-swallowing-breathing; SS, sucking-swallowing.

${ }^{a)}$ Short burst of 3 to 5 sucks, ${ }^{\text {b) }}$ medium burst of 5 to 10 sucks, ${ }^{\text {c) }}$ long burst of 10 to 30 sucks, ${ }^{\text {d) }}$ nutritive sucking repeated more than 10 times without breathing, ${ }^{\mathrm{e})}$ frequent breathing with alternation of longer sucking bursts with desaturation and shorter bursts with stable saturation, ${ }^{\mathrm{f})}$ ratio of SSB is 2-3:1:1 with 10-20 sucking bursts and mild incoordination, ${ }^{\mathrm{g})}$ ratio of SSB is 1-2:1:1 with 20-30 sucking bursts, ${ }^{\text {h) }}$ the rhythm of feeding and swallowing is regular.

while a mature sucking pattern involves a long burst of 10 to 30 sucks. The developmental stages of SSB are classified as previously mentioned in BROSS. The obligatory phase involves nutritive sucking repeated more than 10 times without breathing. The alternating phase involves frequent breathing, but longer sucking bursts that lack saturation are alternated with shorter bursts with stable saturation. In the intermittent phase, the ratio of SSB is 2 to $3: 1: 1$, with 10 - to 20 -suck bursts and mild incoordination. In the coordinated phase, the ratio of SSB is 1 to 2:1:1 with 20 - to 30 -suck bursts. In the integrated phase, the rhythm of feeding and swallowing is regular.

In the FSSPI, we subdivided the framework of six consecutive developmental levels of feeding in BROSS. First, the stage in which even non-nutritive sucking cannot be performed was added. Second, before the obligatory phase, the stage in which nutritive sucking can be performed was included. Third, the alternative phase was subdivided in two stages according to the sucking pattern because the number of sucking bursts increases with the development of feeding and swallowing skills [9]. Thus, the scale score is represented by a 9 -point scale from 0 to 8. A higher score indicates poor feeding and swallowing skills. Clinicians take 10 to 15 minutes to evaluate the VFSS findings of the infant using this scale.

\section{Subjects}

One hundred thirty premature infants (gestational age $<34$ weeks) with a corrected age of less than 3 months who had undergone VFSS at the outpatient clinic or during inpatient consultation, between January 2008 and May 2014, were included in this study. Infants with craniofacial or oral cavity malformations, known clinical syndromes (for example, Down syndrome, etc.), and grade III or IV intraventricular hemorrhage or periventricular leukomalacia were excluded. Intraventricular hemorrhage or periventricular leukomalacia was confirmed by brain ultrasound or magnetic resonance imaging. Data were collected by a physiatrist and they included gestational age (GA) at birth, birth weight, corrected age (CA) at the time of performing VFSS, changes in body weight, and CA at the time of achieving full oral feeding.

\section{Protocol for VFSS}

VFSSs were conducted as described by Logemann [10] with some modifications $[11,12]$. To evaluate infants, the fluoroscope table was tilted vertically and a feeder seat was placed on the ledge in a semi-reclined position at approximately $45^{\circ}$. Careful attention was paid to the stability of the positioning of the infant on the chair or footplate. Images of the swallowing process were taken in the lateral view (and occasionally in the anteroposterior 
view) using fluoroscopic equipment Shimavision 3200 HG (Shimadzu, Kyoto, Japan). In infants who were fed only milk, VFSSs were performed with bottle-feeding by mixing an undiluted liquid barium solution, Solotop solution 140 barium sulfate (Taejoon Pharm, Seoul, Korea) into the milk. The ratio of milk and barium solution was set at 2:1. The infants' own bottles and nipples were used. To monitor radiation exposure during examination, Dose Area Product (DAP) meter PD8100 (Toreck, Yokohama, Japan) was used. Radiation exposure was maintained at $400 \mu \mathrm{Gycm}^{2}$ or less per examination based on the criteria of the Health Protection Agency (HPA), 2005. To minimize radiation exposure, examination time was limited to 2 minutes. Also, collimation of radiation field and pulsed radiation exposure technique were applied. VFSS was carried out when the pulse oximeter N-560 (Nellcor, Minneapolis, MN, USA) had been applied. Tolerance of oxygen saturation and heart rate were reduced to $90 \%$ and baseline heart rate $\pm 10 \%$ of baseline heart rate, respectively.

\section{Verification of reliability of the FSSPI}

For the verification of intra-rater reliability, one physiatrist and three speech-language pathologists with more than 5 years of experience in VFSS evaluated 18 randomly extracted VFSS videos. The same videos were evaluated again 2 weeks later. For the verification of inter-rater reliability, four evaluators assessed these 18 randomly extracted VFSS videos.

\section{Verification of validity of the FSSPI}

For the verification of validity, the correlation between the FSSPI and clinical indicators that reflect the maturation of feeding and swallowing, such as GA at birth, birth weight, CA at the time of performing VFSS, changes in body weight, and CA at the time of achieving full oral feeding, was analyzed $[13,14]$.

\section{Statistics}

The Cronbach's alpha coefficient was calculated to examine the reliability of the FSSPI. To examine the normality of clinical indicators, we applied the Shapiro-Wilk normality test and the Kolmogorov-Smirnov normality test. For the verification of validity, we used the Pearson correlation coefficient in parametric analysis and the Spearman correlation coefficient in nonparametric anal- ysis. The level of significance was set at $\mathrm{p}<0.05$. All analyses were performed using SPSS ver. 23.0 (IBM, Armonk, NY, USA).

\section{RESULTS}

\section{Demographic characteristics}

Eighty-three infants were included in the final analysis. Forty-seven infants who did not meet the inclusion criteria were excluded: 8 infants had a craniofacial or oral cavity malformation, 6 infants were suspected of having a clinical syndrome, and 33 infants had grade III or IV intraventricular hemorrhage or periventricular leukomalacia. Of the 83 infants included, 45 were boys and 38 were girls. The mean GA was $27.3 \pm 2.8$ weeks. The mean body weight at birth was $1.1 \pm 0.4 \mathrm{~kg}$. The mean CA at the time of performing VFSS was $2.1 \pm 54.4$ days and the mean chronological age when VFSS was performed was 3.6 \pm 1.9 months. The corrected age at the time of achieving full oral feeding was recorded in 51 out of the 83 infants (mean age, $1.3 \pm 0.7$ months) (Table 2 ). Information on weight gain was available in 23 infants.

\section{Intra-rater and inter-rater reliability of the FSSPI}

The FSSPI showed a high degree of intra-rater reliability (Cronbach's alpha coefficient $=0.963, \mathrm{p}<0.01$ ) and interrater reliability (Cronbach's alpha coefficient $=0.970$, $\mathrm{p}<0.01)$.

Table 2. Characteristics of subjects $(n=83)$

\begin{tabular}{|c|c|}
\hline Characteristic & Value \\
\hline \multicolumn{2}{|l|}{ Sex } \\
\hline Boy & 45 \\
\hline Girl & 38 \\
\hline Gestational age (week) & $27.3 \pm 2.8$ \\
\hline $\begin{array}{l}\text { Corrected age when VFSS was } \\
\text { performed (day) }\end{array}$ & $2.1 \pm 54.4$ \\
\hline $\begin{array}{l}\text { Chronologic age when VFSS was } \\
\text { performed (month) }\end{array}$ & $3.6 \pm 1.9$ \\
\hline Birth weight $(\mathrm{kg})$ & $1.1 \pm 0.4$ \\
\hline $\begin{array}{l}\text { Corrected age when full oral feeding } \\
\text { was achieved (month) }\end{array}$ & $1.3 \pm 0.7$ \\
\hline
\end{tabular}

Values are presented as number or mean \pm standard deviation.

VFSS, videofluoroscopic swallowing study. 


\section{Validity of the FSSPI}

There was a significant negative correlation between the FSSPI scale score and CA at the time of performing VFSS (Spearman correlation coefficient $=-0.706, p<0.01$ ) (Fig. 1A). Further, a significant positive correlation was observed between the FSSPI scale score and CA at the time of achieving full oral feeding (Pearson correlation coefficient $=0.514, p<0.01$ ) (Fig. 1B). Additionally, there was a significant negative correlation between the FSSPI scale score and weight gain: between the 1st and 2nd month after birth (Pearson correlation coefficient $=-0.437$, p <0.05) (Fig. 2A) and between the 2nd and 3rd month after birth (Pearson correlation coefficient $=-0.432, \mathrm{p}<0.05$ ) (Fig. 2B), respectively.

\section{DISCUSSION}

In this study, we proposed a new clinical scale using VFSS to reflect the development of feeding and swallowing skills in preterm infants. Further, we verified the reliability and validity of the scale. Understanding the development of feeding and swallowing skills is extremely
(A)

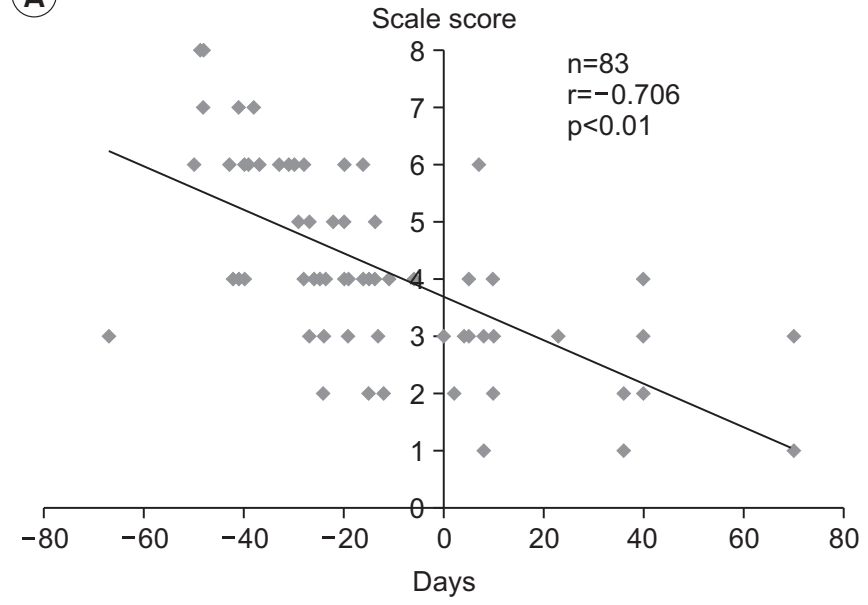

(B)

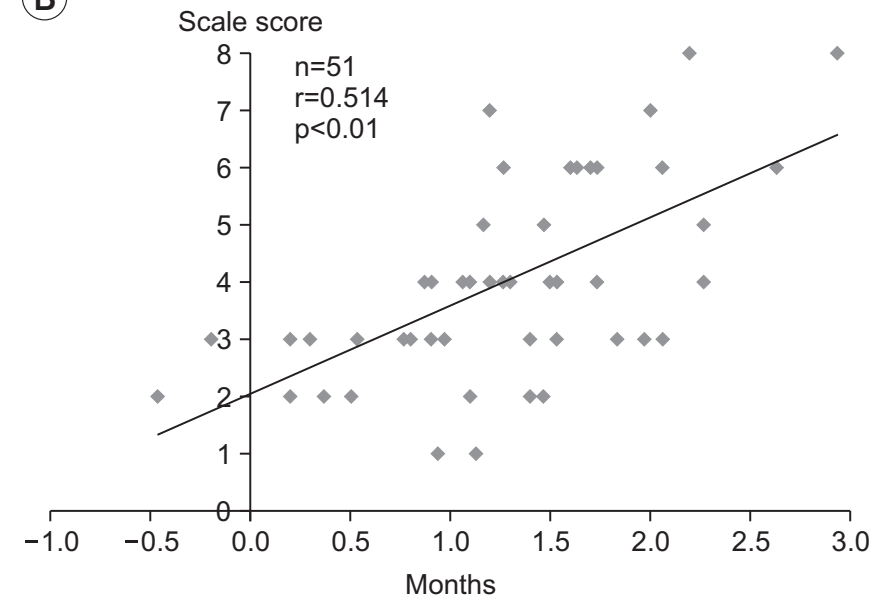

Fig. 1. (A) Correlation between FSSPI scale score and corrected age at the time of performing VFSS. (B) Correlation between FSSPI scale score and chronological age at the time of achieving full oral feeding. FSSPI, Feeding and Swallowing Scale for Premature Infants; VFSS, videofluoroscopic swallowing study.

(A)

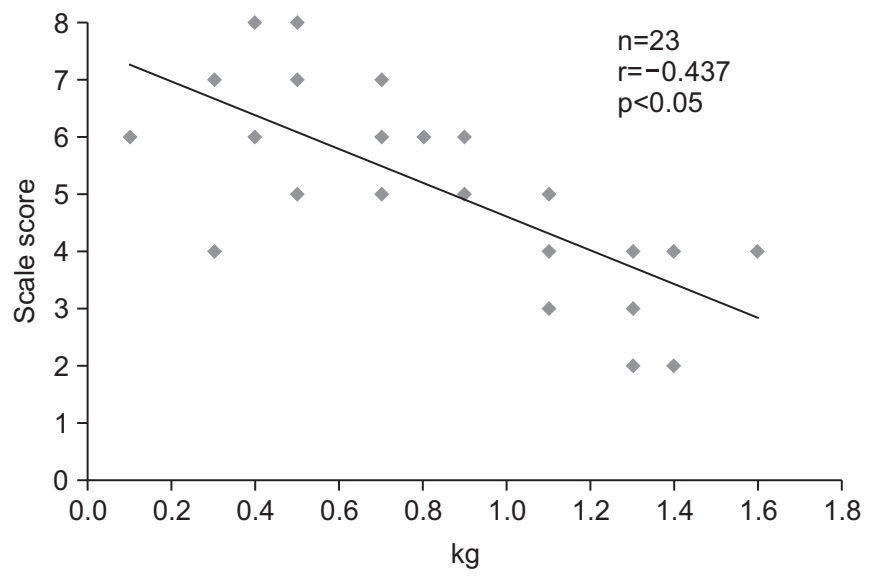

(B)

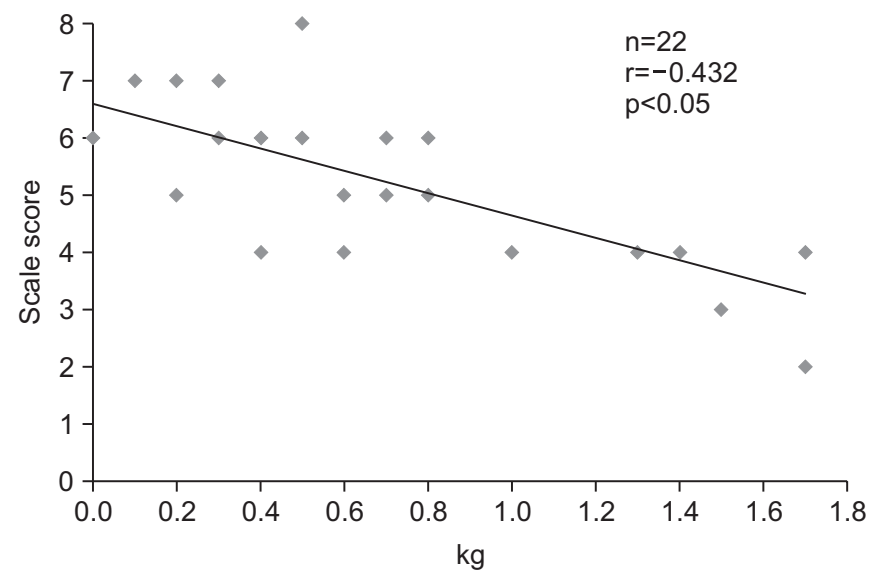

Fig. 2. (A) Correlation between FSSPI scale score and weight gain between the first and second month after birth. (B) Correlation between FSSPI scale score and weight gain between the second and third month after birth. SSPI, Feeding and Swallowing Scale for Premature Infants. 
important in preterm infants as many previous reports have indicated that the feeding and swallowing skills of premature infants are very delayed compared to those of term infants $[15,16]$. VFSS is considered useful to understand the pathophysiologic mechanisms of dysphagia in infancy, such as aspiration, penetration, reflux, insufficient laryngeal excursion, opening of the upper esophageal sphincter, residue, delayed oral/pharyngeal transit time, and/or anatomic defects such as tracheoesophageal fistula and esophageal atresia $[17,18]$. Besides this qualitative information, the FSSPI can provide additional information on the development of feeding and swallowing skills in preterm infants.

There are several clinical scales for evaluating feeding and swallowing function in infants, such as the Schedule for Oral-Motor Assessment (SOMA), the NOMAS, and the Early Feeding Skills (EFS) assessment. SOMA is a standardized procedure for the assessment of oral motor skills in infants aged between 8 and 24 months postnatal [19]. However, there is a limitation in evaluating the early feeding skills of infants younger than 6 months [20]. The EFS assessment was designed to standardize the measurement of the feeding skills of preterm infants and to facilitate the development of individualized interventions to support their skill level. The method of scoring was not available in the medical reports [21]. The NOMAS is a reliable feeding assessment tool for the evaluation of neonatal sucking patterns in pre-term and term infants. The NOMAS provides a description of the infant's feeding patterns and enables the examiner to identify normal oral-motor patterns and to differentiate disorganized from dysfunctional patterns. The oral-motor components in the checklist include rate, rhythmicity, and consistency of the degree of jaw excursion, the direction, range of motion, and timing of tongue movements, and tongue configuration [22]. No numerical scoring method was used to administer and interpret the results [21].

The FSSPI has a clinical strength compared to the above-mentioned scales, as it reflects the early feeding and swallowing development skills of preterm infants (below a corrected age of 3 months). As the FSSPI showed a high correlation with early achievement of full oral feeding and weight gain in early infancy, this scale might be useful to predict early feeding and swallowing outcomes in preterm infants. Further, an individualized feeding intervention to support the skill level of infants could be facilitated based on the development of feeding and swallowing skills in premature infants [23]. For example, for infants with an FSSPI score of 8 or 7 , nonnutritive sucking is recommended using supporting stabilization of body position and behavioral state. Gavage feeding with a positive oral experience and smell should be introduced. For infants with FSSPI scores of 5 to 3 , paced bottle feeding should be used with gavage feeding. The amount of oral feeding is expected to increase as infants learn how to coordinate SSB pattern. Further, infants with an FSSPI score of 2 or 1 are expected to regulate feeding by themselves. If they continuously have feeding-related problems (signs of aspiration, coughing, desaturation and apnea), other potential causes of dysphagia need to be considered.

Our study has several limitations as it was based on a retrospective chart review. Further prospective study is needed to determine whether the FSSPI has a predictive value for feeding outcomes in preterm infants. In this retrospective study with limited data, we found a significant positive correlation between the scale score and CA at the time of achieving full oral feeding. A significant negative correlation between the scale score and weight gain in earlier months was also noted. Premature infants who had craniofacial or oral cavity malformations and a previous history of grade III to IV intracranial hemorrhage or periventricular leukomalacia were excluded from this study because we wanted to evaluate the normal developmental level of oral feeding in premature infants. Further study is needed to verify the criterion validity of the FSSPI through which this scale can differentiate between normally developing preterm infants and infants who develop severe feeding and swallowing complications.

\section{CONFLICT OF INTEREST}

No potential conflict of interest relevant to this article was reported.

\section{REFERENCES}

1. Gewolb IH, Bosma JF, Taciak VL, Vice FL. Abnormal developmental patterns of suck and swallow rhythms during feeding in preterm infants with bronchopulmonary dysplasia. Dev Med Child Neurol 2001;43:4549. 
2. Amaizu N, Shulman R, Schanler R, Lau C. Maturation of oral feeding skills in preterm infants. Acta Paediatr 2008;97:61-7.

3. Goldfield EC, Buonomo C, Fletcher K, Perez J, Margetts S, Hansen A, et al. Premature infant swallowing: patterns of tongue-soft palate coordination based upon videofluoroscopy. Infant Behav Dev 2010;33: 209-18.

4. Williamson LR. Early feeding skills assessment in preterm infants [dissertation]. Lawrence: University of Kansas; 2013.

5. Thoyre SM, Shaker CS, Pridham KF. The early feeding skills assessment for preterm infants. Neonatal Netw 2005;24:7-16.

6. Langmore SE, Schatz K, Olson N. Endoscopic and videofluoroscopic evaluations of swallowing and aspiration. Ann Otol Rhinol Laryngol 1991;100:678-81.

7. Mercado-Deane MG, Burton EM, Harlow SA, Glover AS, Deane DA, Guill MF, et al. Swallowing dysfunction in infants less than 1 year of age. Pediatr Radiol 2001; 31:423-8.

8. Browne JV, Ross ES. Eating as a neurodevelopmental process for high-risk newborns. Clin Perinatol 2011; 38:731-43.

9. Law-Morstatt L, Judd DM, Snyder P, Baier RJ, Dhanireddy $\mathrm{R}$. Pacing as a treatment technique for transitional sucking patterns. J Perinatol 2003;23:483-8.

10. Logemann JA. Evaluation and treatment of swallowing disorders. 2nd ed. San Diego: College Hill Press; 1998. p. 168-80.

11. Uhm KE, Yi SH, Chang HJ, Cheon HJ, Kwon JY. Videofluoroscopic swallowing study findings in full-term and preterm infants with Dysphagia. Ann Rehabil Med 2013;37:175-82.

12. Ko MJ, Kang MJ, Ko KJ, Ki YO, Chang HJ, Kwon JY. Clinical usefulness of Schedule for Oral-Motor Assessment (SOMA) in children with dysphagia. Ann Rehabil Med 2011;35:477-84.
13. Marofi M, Abedini F, Mohammadizadeh M, Talakoub S. Effect of palady and cup feeding on premature neonates' weight gain and reaching full oral feeding time interval. Iran J Nurs Midwifery Res 2016;21:202-6.

14. Rocha AD, Moreira ME, Pimenta HP, Ramos JR, Lucena SL. A randomized study of the efficacy of sensorymotor-oral stimulation and non-nutritive sucking in very low birthweight infant. Early Hum Dev 2007;83: 385-8.

15. Gewolb IH, Vice FL, Schwietzer-Kenney EL, Taciak VL, Bosma JF. Developmental patterns of rhythmic suck and swallow in preterm infants. Dev Med Child Neurol 2001;43:22-7.

16. Lau C, Smith EO, Schanler RJ. Coordination of suckswallow and swallow respiration in preterm infants. Acta Paediatr 2003;92:721-7.

17. Joan CA, Linda B. Pediatric swallowing and feeding. 2nd ed. Albany: Singular; 2001 p. 355-76.

18. Jeong JY, Wang KC, Bang MS. Introduction to cerebral palsy. Seoul: Koonja; 2013. p. 209-13.

19. Reilly S, Skuse D, Mathisen B, Wolke D. The object rating of oral-motor functions during feeding. Dysphagia 1995;10:117-91.

20. Skuse D, Stevenson J, Reilly S, Mathisen B. Schedule for oral-motor assessment (SOMA): methods of validation. Dysphagia 1995;10:192-202.

21. Howe TH, Lin KC, Fu CP, Su CT, Hsieh CL. A review of psychometric properties of feeding assessment tools used in neonates. J Obstet Gynecol Neonatal Nurs 2008;37:338-49.

22. Braun MA, Palmer MM. A pilot study of oral-motor dysfunction in "at-risk" infants. Phys Occup Ther Pediatr 1986;5:13-25.

23. Philbin MK, Ross ES. The SOFFI Reference Guide: text, algorithms, and appendices: a manualized method for quality bottle-feedings. J Perinat Neonatal Nurs 2011;25:360-80. 